\title{
Functional catastrophe analysis of collapse mechanisms for deep tunnels based on the Hoek-Brown failure criterion"
}

\author{
Cheng-ping ZHANG ${ }^{\dagger 1,2}$, Kai-hang HAN ${ }^{1,2}$, Qian FANG ${ }^{1,2}$, Ding-li ZHANG ${ }^{1,2}$ \\ $\left({ }^{1}\right.$ MOE Key Lab of Urban Underground Engineering, Beijing Jiaotong University, Beijing 100044, China) \\ $\left({ }^{2}\right.$ School of Civil Engineering, Beijing Jiaotong University, Beijing 100044, China) \\ ${ }^{\dagger}$ E-mail: chpzhang@bjtu.edu.cn
}

Received Jan. 6, 2014; Revision accepted July 20, 2014; Crosschecked Aug. 26, 2014

\begin{abstract}
A reliable prediction of the characteristics of roof collapse in deep tunnels is still one of the most important and challenging tasks in tunnel engineering. To investigate the collapse mechanisms and possible shapes of the collapsing blocks in deep tunnels, an analytical solution of shape curves for collapsing blocks is derived based on the nonlinear Hoek-Brown failure criterion by using the functional catastrophe theory. The obtained formulas are extremely simple. Furthermore, a judging criterion is proposed to distinguish whether the roof collapses of deep tunnels will occur or not. The effects of rock mass parameters used in the proposed method on the collapsing block shapes of deep tunnels are also discussed. The proposed analytical solution is verified by both the empirical method and the model test.
\end{abstract}

Key words: Deep tunnel, Tunnel roof collapse, Functional catastrophe theory, Hoek-Brown failure criterion doi: $10.1631 /$ jzus.A1400014

Document code: A

CLC number: TU921

\section{Introduction}

The collapse of a tunnel roof, which could cause a lot of harm to human life and property, is a major challenge in tunnel engineering. Due to the inherent uncertainties of rock mass in nature, the precise prediction of tunnel roof collapses is very complex. Terzaghi (1946), who first used the stress-transfer theory to analyze the characteristics of tunnel collapses, also developed a system to estimate the degree of tunnel safety. After Terzaghi, many researchers have extensively investigated the mechanisms of tunnel collapses by using the upper-bound theory (Fraldi and Guarracino, 2009; 2010; 2011; Huang and Yang, 2011; Huang et al., 2012; Fraldi et al., 2014).

\footnotetext{
* Project supported by the National Natural Science Foundation of China (Nos. 51008015 and 51378002), the Fundamental Research Funds for the Central Universities (No. 2012JBZ013), and the Program for New Century Excellent Talents in University (No. NCET-12-0770), China

(c) Zhejiang University and Springer-Verlag Berlin Heidelberg 2014
}

The catastrophe theory, found by Thom (1972), provides an innovative approach to study the discontinuous phenomena. This theory has been proved to be effective in analyzing the stability problems in geology and geomechanics. Henley (1976) used the catastrophe theory to analyze the phenomena in geology and some examples of its usages in geology (e.g., volcanology, sedimentology, and structural geology) were presented. Pan et al. (2006) established a fold catastrophe model of tunnel rock burst and reported that the occurrences of rock burst are related to both the ratio of elastic modulus to descendent modulus of the rock mass and the rock mass crack growth degree. Based on the fold catastrophe theory, Miao et al. (2009) presented a model of the seepage flow system to analyze the dynamical behavior of water or gas flows in damaged rock, and the influences of different key parameters on the stability of seepage flow systems were obtained. With the catastrophe theory and the discontinuous deformation analysis (DDA) method, Xia et al. (2012) studied the stability of tunnel surrounding rock and obtained the 
safety factors for tunnels, which can be adopted in practical projects to guarantee the safety of the tunnels. Tao et al. (2013) studied the mid-long-term landslide evolution across a low-latitude highland area in China by using the cusp catastrophe theory and determined the influence of human activities on landslide intensities. Ren et al. (2013) proposed a cusp catastrophe model to analyze the potential damage mode of the surrounding rock in the Gonghe tunnel, China and the failure criterion of the surrounding rock was jointly derived by using the discrete element method (DEM).

The current research on tunnel collapse is generally based on the elementary catastrophe theory (ECT). In the ECT, the degenerate critical point of the total potential (one of seven elementary functions of state variable $x$ ) of the studied system is obtained by analyzing the first- and second-order partial derivatives of the total potential. Then the critical value $x_{\mathrm{c}}$ of the state variable is obtained when the catastrophe occurs. However, in a complex system, the total potential of the system often has a complex mathematical form (such as a functional of the state function $f(x))$ rather than the elementary functions. Du (1994) proposed the functional catastrophe theory (FCT), of which the total potential is employed in the form of a functional instead of an elementary function, and he successfully solved some complex physical and economic problems with the FCT. In the FCT, it requires to obtain the degenerate critical point of the total potential by analyzing the first- and second-order variations of the total potential. In a similar way, the critical value $f_{\mathrm{c}}(x)$ of the state variable is obtained when catastrophe occurs. To investigate the shape curve $f(x)$ of the collapsing blocks in deep tunnels by using the catastrophe theory, the potential of the studied system will be a functional based on $f(x)$. Therefore, the FCT can be used to analyze tunnel stability problems.

In this study, the detaching zone of a collapsing block is the studied system. Referring to the Hoek-Brown failure criterion, the potential of the studied system is first established. Then the FCT is employed to investigate the mechanisms of tunnel roof collapse. The analytical solution of the collapsing block shape curve in deep tunnels is obtained and the judging criterion is put forward to distinguish whether the roof collapse of deep tunnels will occur or not. The effects of different parameters on the collapsing block shape are also discussed. Moreover, the proposed analytical solution is verified by both the empirical method and the model test.

\section{Functional catastrophe theory}

\subsection{Catastrophic conditions for the functional}

There are seven fundamental models of ECT which have been summarized by Thom (1972). The seven elementary functions are defined by polynomial functions, as shown in Table 1. According to the ECT, Eqs. (1) and (2) are used to judge the degenerate critical point of the potential. It means that the potential in the critical points of Eqs. (1) and (2) is structurally unstable, i.e., a catastrophe is going to occur.

$$
\begin{gathered}
D V=0, \\
\operatorname{det}(H V)=0,
\end{gathered}
$$

where $D V$ and $\operatorname{det}(H V)$ are the differential vector and the determinant of Hessian matrix of function $V[x, y]$, respectively.

Table 1 Potential functions used in elementary catastrophe theory

\begin{tabular}{ll}
\hline \multicolumn{1}{c}{ Name } & \multicolumn{1}{c}{ Potential function } \\
\hline Fold & $x^{3} / 3+a x$ \\
Cusp & $x^{4} / 4+a x^{2} / 2+b x$ \\
Swallowtail & $x^{5} / 5+a x^{3} / 3+b x^{2} / 2+c x$ \\
Butterfly & $x^{6} / 6+a x^{4} / 4+b x^{3} / 3+c x^{2} / 2+d x$ \\
Elliptic umbilic & $x^{3}-3 x y^{2}+a\left(x^{2}+y^{2}\right)+b x+c y$ \\
Hyperbolic umbilic & $x^{3}+y^{3}+a x y+b x+c y$ \\
Parabolic umbilic & $x^{2} y+y^{4}+a x^{2}+b y^{2}+c x+d y$ \\
\hline
\end{tabular}

If the total potential of the studied system is defined by a functional (Eq. (3)), it will be a challenge to determine the catastrophe occurrence of the studied system.

$$
J[f(x)]=\int_{a}^{b} F\left[x, f(x), f^{\prime}(x)\right] \mathrm{d} x,
$$

where the prime indicates the derivative of the function with respect to its variable, i.e., $f^{\prime}(x)=\partial f(x) / \partial x$.

$\mathrm{Du}$ (1994) derived the necessary and sufficient conditions for a functional catastrophe, which are shown in Eqs. (4) and (5). They are formally similar with Eqs. (1) and (2), respectively. 


$$
\begin{gathered}
\delta J=0, \\
\delta^{2} J=0,
\end{gathered}
$$

where $\delta J$ and $\delta^{2} J$ are the first- and second-order variations of functional $J f f(x)]$, respectively.

According to Eqs. (3)-(5), the catastrophic conditions for functional $J[f(x)]$ can be written as

$$
\begin{gathered}
\delta J=\int_{a}^{b}\left(\frac{\partial F}{\partial f(x)} \delta f(x)+\frac{\partial F}{\partial f^{\prime}(x)} \delta f^{\prime}(x)\right) \mathrm{d} x=0 \\
\delta^{2} J=\int_{a}^{b}\left(\frac{\partial^{2} F}{\partial f(x)^{2}} \delta f(x)^{2}+2 \frac{\partial^{2} F}{\partial f(x) \partial f^{\prime}(x)} \delta f(x) \delta f^{\prime}(x)\right. \\
\left.+\frac{\partial^{2} F}{\partial f^{\prime}(x)^{2}} \delta f^{\prime}(x)^{2}\right) \mathrm{d} x=0 .
\end{gathered}
$$

With the help of a subsection integral, the two specific forms of the catastrophic conditions for the functional are obtained:

$$
\begin{gathered}
\frac{\partial F}{\partial f(x)}-\frac{\partial}{\partial x}\left(\frac{\partial F}{\partial f^{\prime}(x)}\right)=0 \\
\frac{\partial^{2} F}{\partial f(x)^{2}}-2 \frac{\partial}{\partial x}\left(\frac{\partial^{2} F}{\partial f(x) \partial f^{\prime}(x)}\right)+\frac{\partial^{2}}{\partial x^{2}}\left(\frac{\partial^{2} F}{\partial f^{\prime}(x)^{2}}\right)=0 .
\end{gathered}
$$

\subsection{Basic solution procedure for FCT to solve the problem of tunnel collapse}

Firstly, it is important to obtain the total potential of the studied system $J\left[x, f(x), f^{\prime}(x)\right]$, and then to obtain the $F\left[x, f(x), f^{\prime}(x)\right]$. Note that $f(x)$ is a function describing the outline of the collapsing block.

Secondly, using Eqs. (8) and (9), a group of differential equations about $f(x)$ can be obtained. By integrating the differential equations, the shape curve $(f(x))$ of the collapsing block can be obtained.

Finally, the unknown constants in $f(x)$ can be determined by boundary transversality conditions and geometric compatibility equations.

\section{Catastrophe state analysis of a deep tun- nel collapse}

The estimation of the roof stability of deep tunnels primarily lies in determining the shape and dimension of the collapsing blocks which can actually collapse from the roof of the tunnel. By using the upper-bound theorem, Fraldi and Guarracino (2010) proposed a mechanical model for deep tunnels and obtained the upper solution of collapsing block shapes based on the Hoek-Brown failure criterion. The aim of this study is to analyze the mechanisms and the shape of deep tunnel collapses by using another method, FCT. The model assumptions are the same as Fraldi and Guarracino (2010). So the model can be used to analyze the problem presented in this study. A subtle difference in Fig. 1 with the original mechanical model is that the velocity $\dot{u}$ is used instead of displacement $u$. The reason for the difference is that the research object of the FCT and the upperbound theorem are the total potential and total dissipation of the studied system, respectively.

To solve the proposed problem by using the catastrophe theory, some assumptions are made. In this study, only the gravity field is considered, regardless of the tectonic stress field, which is consistent with the relative reference (Fraldi and Guarracino, 2010). The behavior of the rock mass is elastic-perfect plastic. The yield surface is convex and the plastic deformation rate can be obtained from the yield function through an associated flow rule. The changes in the geometry of the collapsing block can be regarded as insignificant through the onset of the collapse (rigidplastic behavior). The problem can be considered plane. The Hoek-Brown failure criterion proposed by Hoek and Brown (1980) is adopted for the rock mass. As the total potential along the failure surface is caused by normal and shear stresses, the Hoek-Brown failure criterion used is expressed as

$$
\tau_{\mathrm{n}}=A \sigma_{\mathrm{c}}\left(\frac{\sigma_{\mathrm{n}}+\sigma_{\mathrm{t}}}{\sigma_{\mathrm{c}}}\right)^{B}, A, B \in(0,1), \sigma_{\mathrm{c}}>\sigma_{\mathrm{t}} \geq 0,(10)
$$

where $\sigma_{\mathrm{n}}$ is the normal stress, $\tau_{\mathrm{n}}$ is the shear stress, $A$ and $B$ are material parameters characterizing the rock mass, $\sigma_{\mathrm{c}}$ and $\sigma_{\mathrm{t}}$ are the uniaxial compressive strength and the tensile strength of the rock mass, respectively.

In Fig. 1, $L$ is the half-width of the collapsing block, $R$ is the tunnel radius, $H$ is the height of the collapsing block, $h$ is the intercept in $y$-axis of $f(x), \rho$ is the weight per unit volume of the rock mass, $w$ is the thickness of the plastic detaching zone, and $g(x)$ is a known function describing the shape of a circular tunnel.

$$
g(x)=-\sqrt{R^{2}-x^{2}} .
$$




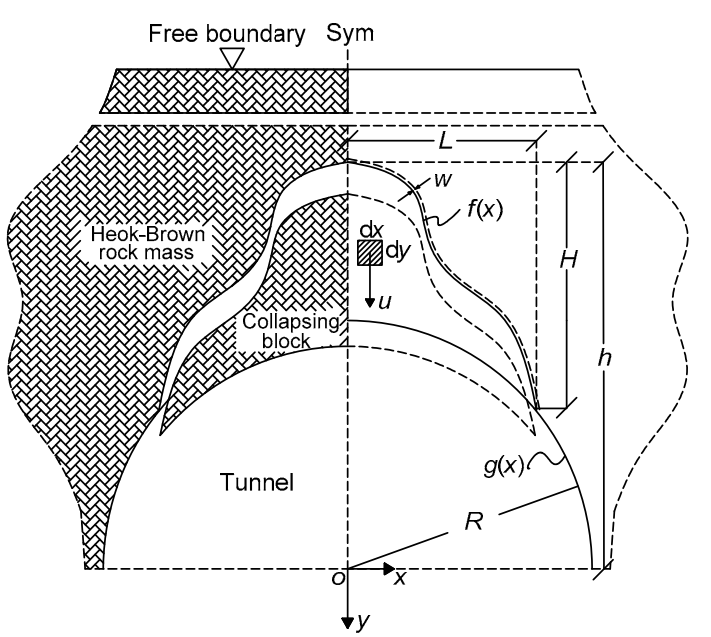

Fig. 1 Collapsing pattern of the deep tunnels

The total potential is obtained by computing the internal forces potential energy in the detaching zone and external loads potential energy on the detaching surface. Referring to the formula presented by Fraldi and Guarracino (2010), the total potential of the studied system consists of two parts, the strain energy of the internal forces on the detaching zone and the work of the applied loads of the detaching surface.

By following a purely geometrical perspective and making reference to Fig. 1, the normal and tangential plastic strain components of detaching surface can be written as

$$
\begin{gathered}
\varepsilon_{\mathrm{n}}=(u / w)\left[1+f^{\prime}(x)^{2}\right]^{-1 / 2}, \\
\gamma_{\mathrm{n}}=-(u / w) f^{\prime}(x)\left[1+f^{\prime}(x)^{2}\right]^{-1 / 2} .
\end{gathered}
$$

At impending collapse, the strain energy $\left(U_{\mathrm{i}}\right)$ of the internal forces on the detaching zone results in

$$
\begin{aligned}
U_{\mathrm{i}}= & \sigma_{\mathrm{n}} \varepsilon_{\mathrm{n}}+\tau_{\mathrm{n}} \gamma_{\mathrm{n}} \\
= & {\left[-\sigma_{\mathrm{t}}+\sigma_{\mathrm{c}}(A B)^{1 /(1-B)}\left(1-B^{-1}\right) f^{\prime}(x)^{1 /(1-B)}\right] } \\
& \times u /\left[w \sqrt{1+f^{\prime}(x)^{2}}\right],
\end{aligned}
$$

while the work of the applied loads per unit length $\left(W_{\mathrm{e}}\right)$ of the detaching surface is

$$
W_{\mathrm{e}}=\rho[f(x)-g(x)] u .
$$

Then, the total potential of the detaching zone caused by normal and shear stresses can be expressed as

$$
\begin{aligned}
& J\left[x, f(x), f^{\prime}(x)\right]=-\int_{0}^{L} \rho[f(x)-g(x)] u \mathrm{~d} x \\
& +\int_{0}^{L}\left[\sigma_{\mathrm{c}}(A B)^{1 /(1-B)}\left(1-B^{-1}\right) f^{\prime}(x)^{1 /(1-B)}-\sigma_{\mathrm{t}}\right] u \mathrm{~d} x .
\end{aligned}
$$

Using the functional catastrophe theory, the function $F$ is

$$
\begin{aligned}
F= & F\left[x, f(x), f^{\prime}(x)\right]=-\rho[f(x)-g(x)] u \\
& +\left[\sigma_{\mathrm{c}}(A B)^{1 /(1-B)}\left(1-B^{-1}\right) f^{\prime}(x)^{1 /(1-B)}-\sigma_{\mathrm{t}}\right] u .
\end{aligned}
$$

The key in the catastrophic state analysis is to find the specific expression of $f(x)$ with the help of Eqs. (8) and (9).

Substituting Eq. (17) into Eqs. (8) and (9), the explicit forms of the group of differential equations of $f(x)$ for the problem are

$$
\begin{gathered}
-\rho-\frac{\mathrm{d}}{\mathrm{d} x}\left(-\sigma_{\mathrm{c}}(A B)^{1 /(1-B)} B^{-1} f^{\prime}(x)^{B /(1-B)}\right)=0 \\
\frac{\mathrm{d}^{2}}{\mathrm{~d} x^{2}}\left(-\sigma_{\mathrm{c}}(A B)^{1 /(1-B)}(1-B)^{-1} f^{\prime}(x)^{(2 B-1) /(1-B)}\right)=0 .
\end{gathered}
$$

Integrating Eq. (18), the detaching curve $f(x)$ is obtained:

$$
f(x)=A^{-1 / B}\left(\rho / \sigma_{\mathrm{c}}\right)^{(1-B) / B}\left(x+\rho^{-1} m\right)^{1 / B}-h,
$$

where $m$ and $h$ are two integration constants.

Substituting Eq. (20) into Eq. (19), we can obtain:

$$
A^{1 / B} \rho^{(2 B-1) / B} \sigma_{\mathrm{c}}^{(1-B) / B}(2 B-1)\left(x+\rho^{-1} m\right)^{-1 / B}=0 .
$$

Eq. (21) is required to be zero for any value of $x$. This means that the value of $B$ must be 0.5 , which is the result of Eq. (5), i.e., one of the catastrophic conditions. In addition, the parameter $B$ determines the power exponent of $f(x)$, so it also describes the shape of the collapsing block. Based on the value of $B$, the reduced form of $f(x)$ can be obtained:

$$
f(x)=A^{-2}\left(\rho / \sigma_{\mathrm{c}}\right)\left(x+\rho^{-1} m\right)^{2}-h .
$$

There are two unknown parameters $m$ and $h$ in Eq. (22), which can be determined by boundary conditions. Obviously, point $A$ moves along the $y$-axis and point $B$ moves along the tunnel boundary as shown in Fig. 2. All the boundary conditions 
mentioned above can be obtained from Wang and Wang (2007). It requires that Eq. (17) should satisfy both Eq. (23) and Eq. (24):

$$
\begin{gathered}
\left.\frac{\partial F}{\partial f^{\prime}(x)}\right|_{x=0}=0, \\
F-\left.\left[f^{\prime}(x)-g^{\prime}(x)\right] \frac{\partial F}{\partial f^{\prime}(x)}\right|_{x=L}=0 .
\end{gathered}
$$

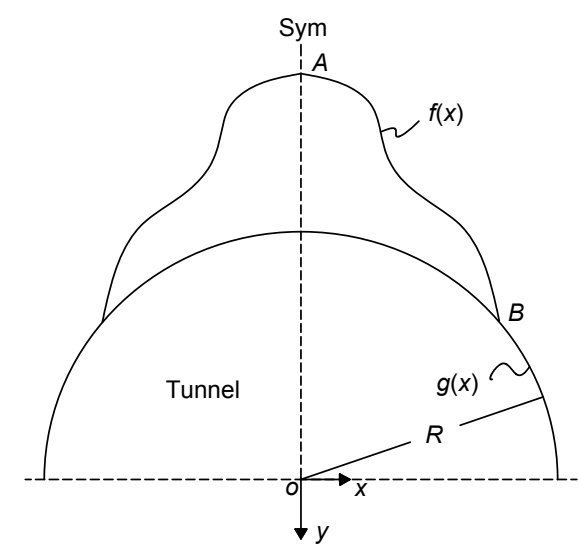

Fig. 2 Boundary conditions of collapsing block

Substituting Eq. (17) into Eqs. (23) and (24), the explicit forms of the transversality conditions are

$$
\begin{aligned}
-\sigma_{\mathrm{c}} & (A B)^{1 /(1-B)} B^{-1} f^{\prime}(0)^{B /(1-B)}=-\sigma_{\mathrm{c}}(A B)^{1 /(1-B)} B^{-1} \\
\times & {\left[A^{-1 / B}\left(\rho / \sigma_{\mathrm{c}}\right)^{(1-B) / B} B^{-1}\left(0+\rho^{-1} m\right)^{(1-B) / B}\right]^{B /(1-B)}=0 } \\
-\sigma_{\mathrm{t}} & +\sigma_{\mathrm{c}}(A B)^{1 /(1-B)}\left(1-B^{-1}\right)\left(k B^{-1} L^{(1-B) / B}\right)^{1 /(1-B)} \\
& -\rho\left(k L^{1 / B}-h+\sqrt{R^{2}-L^{2}}\right) \\
& -\left(k B^{-1} L^{(1-B) / B}-L / \sqrt{R^{2}-L^{2}}\right) \\
& \times\left[-\sigma_{\mathrm{c}}(A B)^{1 /(1-B)} B^{-1}\left(k B^{-1} L^{(1-B) / B}\right)^{B /(1-B)}\right]=0 .
\end{aligned}
$$

Simplifying Eqs. (25) and (26), the values of $m$ and $h$ in the expression of collapsing block are determined:

$$
\begin{aligned}
& m=0, \\
& h=\sigma_{\mathrm{t}} / \rho+\sqrt{R^{2}-L^{2}}+L^{2} / \sqrt{R^{2}-L^{2}} .
\end{aligned}
$$

Substituting the values of $m$ and $h$ into Eq. (22), the shape curve of collapsing block can be obtained:

$$
\begin{aligned}
f(x)= & A^{-2}\left(\rho / \sigma_{\mathrm{c}}\right) x^{2} \\
& -\left(\sigma_{\mathrm{t}} / \rho+\sqrt{R^{2}-L^{2}}+L^{2} / \sqrt{R^{2}-L^{2}}\right) .
\end{aligned}
$$

Now we obtain the curve which describes the shape and dimensions of the collapsing block of deep tunnels. The curve is a parabola with $y$-axis being the axis of symmetry and $h$ being the $y$-intercept.

The value of $L$ is still unknown in Eq. (29). However, on account of its geometrical meaning, $L$ can be easily obtained. It can be seen in Fig. 1 that there is a geometric compatibility condition which needs to be satisfied:

$$
f(x=L)=g(x=L)
$$

Substituting Eqs. (11) and (29) into Eq. (30) results in

$$
A^{-2}\left(\rho / \sigma_{\mathrm{c}}\right) L^{2}-\sigma_{\mathrm{t}} / \rho-L^{2} / \sqrt{R^{2}-L^{2}}=0 .
$$

Then, the value of $L$ can be obtained by solving Eq. (31).

Moreover, it is possible to compute the overall weight of the collapsing block per unit length $(P)$ by

$$
\begin{aligned}
P= & 2 \int_{0}^{L} \rho[g(x)-f(x)] \mathrm{d} x \\
= & -2 \rho\left(\frac{L}{2} \sqrt{R^{2}-L^{2}}+\frac{R^{2}}{2} \arcsin \frac{L}{R}\right)-\frac{2 \rho}{3 A^{2}}\left(\frac{\rho}{\sigma_{\mathrm{c}}}\right) L^{3} \\
& +2 \rho\left(\sigma_{\mathrm{t}} / \rho+\sqrt{R^{2}-L^{2}}+L^{2} / \sqrt{R^{2}-L^{2}}\right) L .
\end{aligned}
$$

\section{Examples and remarks}

\subsection{Judging criterion for a deep tunnel collapse}

In Fig. 1, the height of the collapsing block $H$ can be obtained from Eq. (28):

$$
H=h-\sqrt{R^{2}-L^{2}}=\sigma_{\mathrm{t}} / \rho+L^{2} / \sqrt{R^{2}-L^{2}} .
$$

A tunnel can be regarded as deep buried if the distance between the tunnel crown and the ground surface $H_{\mathrm{ar}}$ satisfies the following inequality:

$$
H_{\text {ar }} \gg H=\sigma_{\mathrm{t}} / \rho+L^{2} / \sqrt{R^{2}-L^{2}} .
$$




\subsection{Influence of different rock mass parameters on the collapsing block shape}

Based on Eq. (29), the shape curve of a collapsing block for deep tunnels can be obtained. The influence of different rock mass parameters on the collapsing block shape is shown in Fig. 3. Note that both the width and the height of the collapsing block tend to decrease with the values of $\sigma_{\mathrm{c}}, \sigma_{\mathrm{t}}$ (Fig. 3a), and $A$ (Fig. 3b). On the contrary, the width and the height of the collapsing block tend to increase with the value of the density $\rho$ (Fig. 3c). A highlight identified is that the collapsing block shape is sensitive to parameter $A$. The higher the values of $\sigma_{\mathrm{c}}, \sigma_{\mathrm{t}}$, and $A$ are or the lower the value of $\rho$ is, the smaller the collapsing blocks are.

Table 2 shows the shape curve of the collapsing blocks for different tunnel radius $R$ varying from 3 to $7 \mathrm{~m}$. The rock mass is characterized by the following parameters: $A=0.15, B=0.5, \rho=25 \mathrm{kN} / \mathrm{m}^{3}, \sigma_{\mathrm{c}}=1.5 \mathrm{MPa}$, and $\sigma_{\mathrm{t}}=0.15 \mathrm{MPa}$. With reference to Table 2 , it is worth noting that the half-width of the collapsing block $(L)$ increases almost at the same rate with the increment of the tunnel radius $(R)$ (when the radius doubles, the increment in the width of the collapsing block is about $127 \%$ ), whereas the height of the collapsing block $(H)$ sharply increases with the increment of the tunnel radius (when the radius doubles, the increment in the height of the collapsing block is more than fivefold). Therefore, the ratio of the height to width $(H /(2 L))$ also increases with the increment of the tunnel radius.

Figs. $4 \mathrm{a}-4 \mathrm{c}$ show the height, width, and weight of the collapsing blocks for different tunnel radius, respectively. For tunnel design, the prediction and mitigation of the damage caused by constructioninduced tunnel collapse and the determination of the overburden on the tunnel lining are major concerns. The results obtained in Fig. 4 can be helpful for tunnel engineers, as they cannot only be used to predict the height and width of the collapsing blocks under unsupported conditions but also give a direct estimate of the overburden on the tunnel lining.

\section{Comparisons}

\subsection{Comparison with the natural arch theory}

The natural arch theory proposed by Protodyakonov (1907) has been widely employed (Meng et al., 2012; Qiu et al., 2012) to determine the range of roof collapses in tunnel engineering. It states that the rock mass above the natural arch is undisturbed while
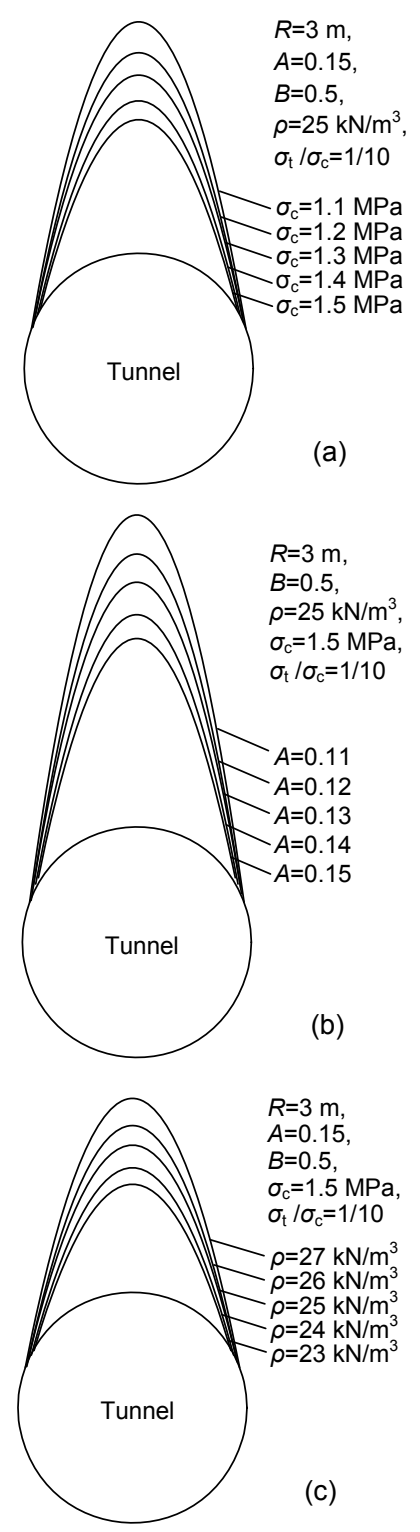

Fig. 3 Shapes of collapsing blocks vs. different rock parameters

(a) Variations of $\sigma_{\mathrm{c}}$ and $\sigma_{\mathrm{t}}$; (b) Variations of $A$; (c) Variations of $\rho$

Table 2 Dimensions of the collapsing blocks vs. tunnel radius

\begin{tabular}{ccrc}
\hline$R(\mathrm{~m})$ & $L(\mathrm{~m})$ & $H(\mathrm{~m})$ & $H /(2 L)$ \\
\hline 3.00 & 2.58 & 3.45 & 0.67 \\
4.00 & 3.73 & 7.76 & 1.04 \\
5.00 & 4.81 & 13.45 & 1.40 \\
6.00 & 5.84 & 20.63 & 1.77 \\
7.00 & 6.86 & 29.28 & 2.13 \\
\hline
\end{tabular}



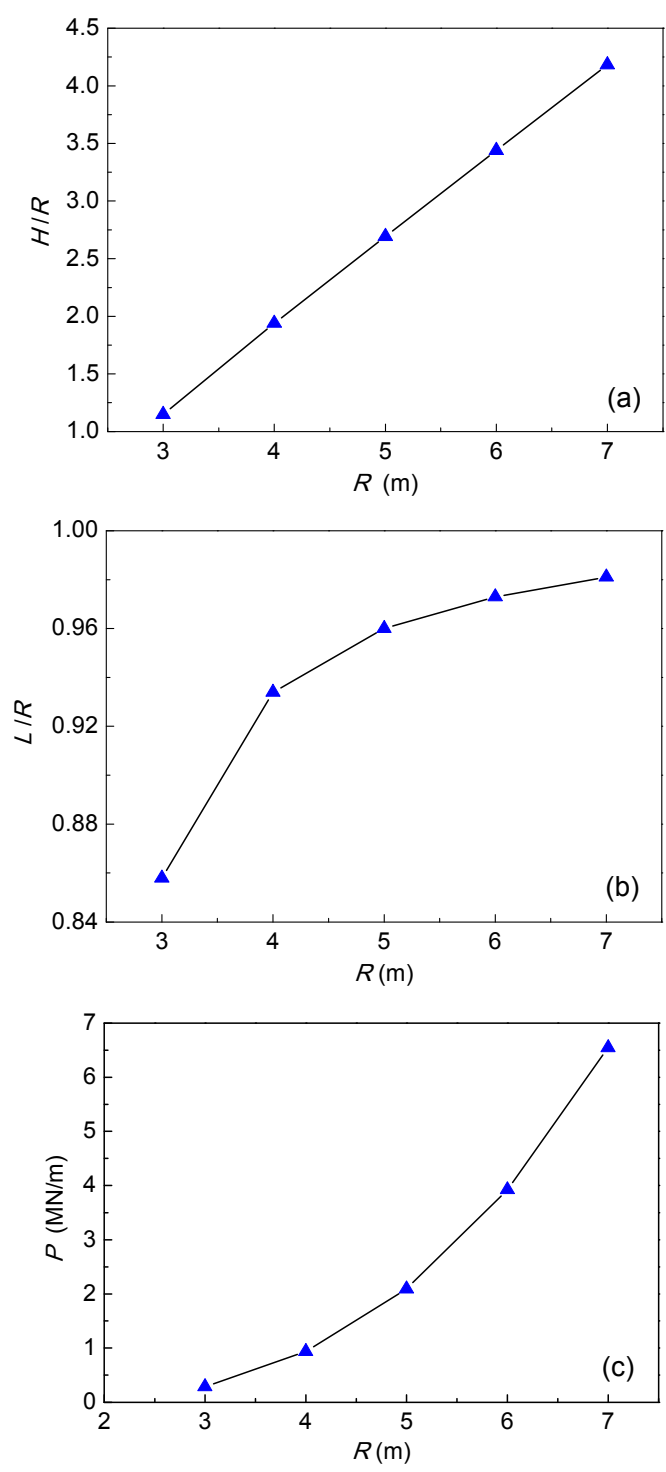

Fig. 4 Variations of $H / R$ (a), $L / R$ (b), and the weight $P$ (c) of the collapsing blocks with respect to tunnel radius $A=0.15, B=0.5, \rho=25 \mathrm{kN} / \mathrm{m}^{3}, \sigma_{\mathrm{c}}=1.5 \mathrm{MPa}, \sigma_{\mathrm{t}} / \sigma_{\mathrm{c}}=1 / 10$

the part below is disturbed to collapse. In Fig. 5, $N$ and $H_{0}$ are the vertical and horizontal pushing forces on the natural arch, respectively, $p_{\mathrm{v}}$ is the vertical uniform load, $h_{\mathrm{na}}$ is the height of the natural arch, and $a$ is the radius of the tunnel. According to the natural arch theory, the natural arch is in a shape of a parabola:

$$
y=\frac{p_{\mathrm{v}}}{2 H_{0}} x^{2} .
$$

Comparing the results obtained by Eqs. (29) and (35), it can be found that the curves are both in the
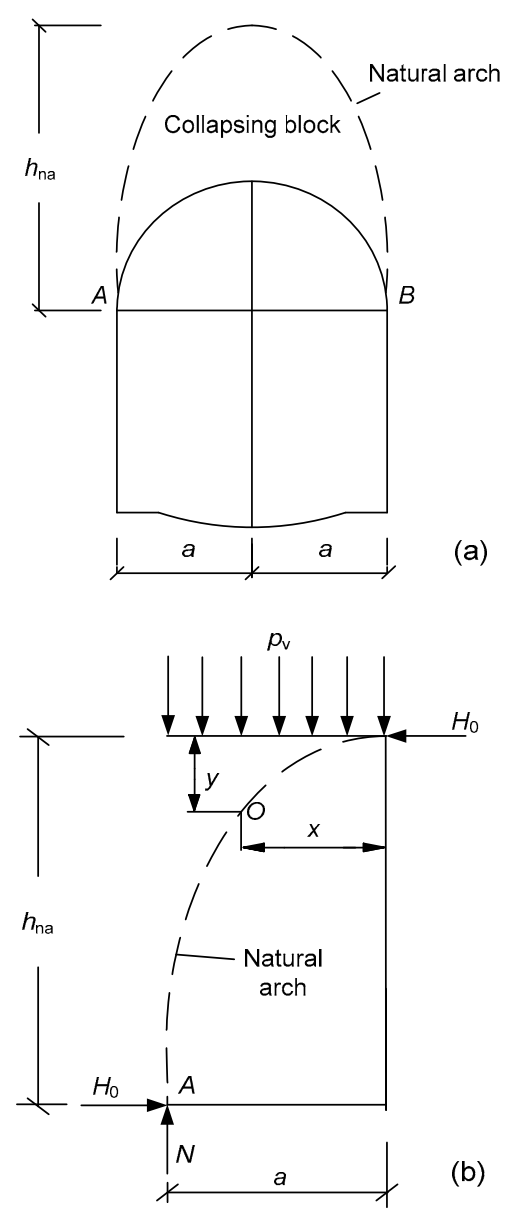

Fig. 5 Calculation model for the natural arch theory (Protodyakonov, 1907)

(a) Sketch map; (b) Mechanical analysis

shape of a parabola. Therefore, the outline of the collapsing block in deep tunnels is in the form of a parabola, which agrees very well with the proposed failure shape according to the natural arch theory.

\subsection{Comparison with the model test}

Li (2013) employed the model test to study the deep tunnel collapse characteristics with a plane strain model test device (Fig. 6). The geometrical similarity ratio of the model tests was $1 / 30$. A type of material, composed of barite, quartz, and vaseline, was selected to represent the surrounding ground. The weight ratio of the ingredients barite:quartz:vaseline was 8.0:5.0:0.6. The tunnel excavation process was modeled by the pressure release of an airbag inside the tunnel. The stress field of the model test was produced by gravity alone. According to Li (2013)'s research, the collapse observed in a model test is 
shown in Fig. 7a. The nonlinear curve fitting of the collapse is shown in Fig. 7b. The results show that the fitting is very good because the adjusted $R^{2}$ is 0.983 , which is nearly equal to 1 . Note that the outline of the collapsing block is in the form of a parabola. The proposed method is thus verified by the laboratory model test.

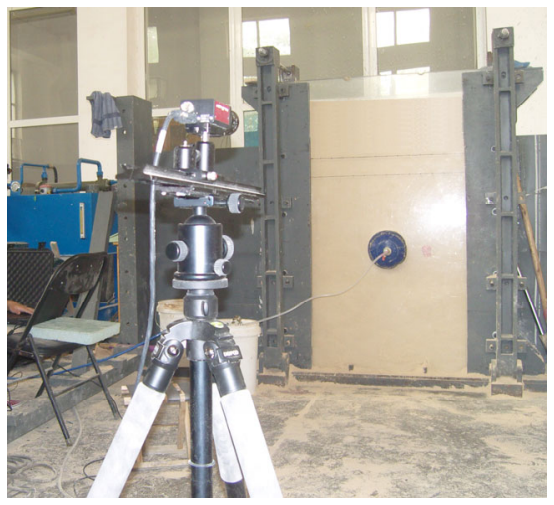

Fig. 6 Model test device (Li, 2013)

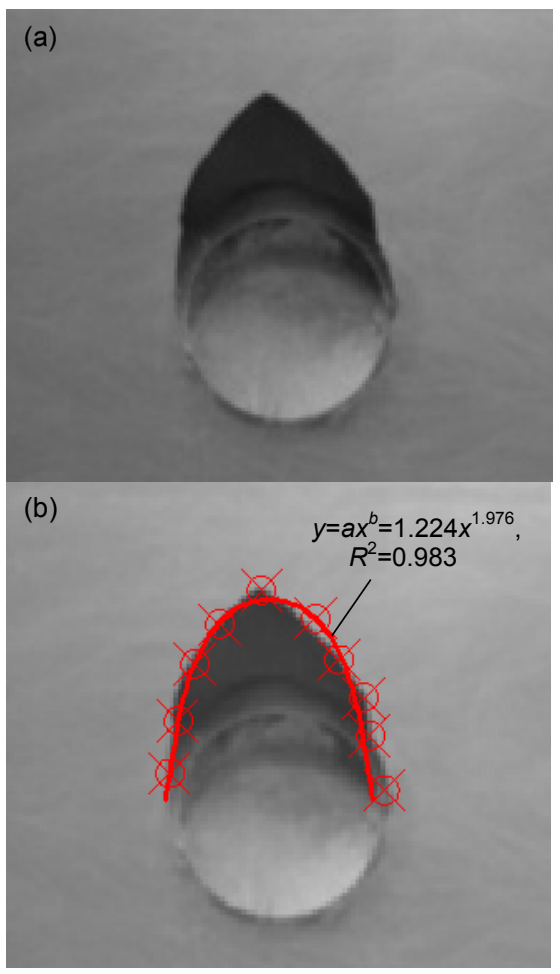

Fig. 7 Nonlinear curve fitting of the collapse observed in a model test

(a) Shape of collapsing block (Li, 2013); (b) Nonlinear curve fitting; the circles with " $\mathrm{X}$ " marked describe the collapsing block boundary; the full line is the result of nonlinear curve fitting

\section{Conclusions}

The potential in the FCT is employed in the form of a functional while the potential in the ECT is employed in the form of an elementary function. The former method greatly excels the latter in solving some complex problems. A major contribution of this paper is that the FCT is the first to investigate the collapse mechanisms and possible collapsing block shapes of deep tunnels. Some conclusions can be drawn as follows:

1. Based on the nonlinear Hoek-Brown failure criterion, an analytical solution of the shape curve for the collapsing blocks of deep tunnels is derived using the FCT. The obtained formulas cannot only be used to predict the height and width of the collapsing block under unsupported conditions but also give a direct estimate of the overburden on the tunnel lining. Due to the simplicity of the obtained formulas, they can be easily used by tunnel engineers and researchers.

2. According to the FCT, based on the nonlinear Hoek-Brown failure criterion, a judging criterion is proposed to distinguish whether the roof collapse of deep tunnels will occur or not, and the outline of the collapsing blocks in deep tunnels is in the form of a parabola. The influences of rock mass parameters on the shape curves of collapsing blocks in deep tunnels are obtained. It is shown that the higher the values of $\sigma_{\mathrm{c}}, \sigma_{\mathrm{t}}$, and $A$ are or the lower the value of $\rho$ is, the smaller the collapsing blocks are.

3. To validate the analytical solution, the results obtained in this paper are compared with those obtained by both an empirical method and a model test. By analyzing the natural arch theory proposed by Protodyakonov (1907) and the results of a plane strain model test, it can also be concluded that the outline of the collapsing blocks is in the form of a parabola. The agreements indicate the validity of the proposed method.

\section{References}

Du, X.F., 1994. Application of Catastrophe Theory in Economic Field. University of Electronic Science and Technology Press, Chengdu, China, p.1-17 (in Chinese).

Fraldi, M., Guarracino, F., 2009. Limit analysis of collapse mechanisms in cavities and tunnels according to the Hoek-Brown failure criterion. International Journal of Rock Mechanics \& Mining Science, 46(4):665-673. [doi:10.1016/j.jirmms.2008.09.014]

Fraldi, M., Guarracino, F., 2010. Analytical solutions for collapse mechanisms in tunnels with arbitrary cross sections. 
International Journal of Solids and Structures, 47(2):216223. [doi:10.1016/j.ijsolstr.2009.09.028]

Fraldi, M., Guarracino, F., 2011. Evaluation of impending collapse in circular tunnels by analytical and numerical approaches. Tunnelling and Underground Space Technology, 26(4):507-516. [doi:10.1016/j.tust.2011.03.003]

Fraldi, M., Gesualdo, A., Guarracino, F., 2014. Influence of actual plastic hinge placement on the behavior of ductile frames. Journal of Zhejiang University-SCIENCE A (Applied Physics \& Engineering), 15(7):482-495. [doi:10. 1631/jzus.A1400031]

Henley, S., 1976. Catastrophe theory models in geology. Journal of the International Association for Mathematical Geology, 8(6):649-655. [doi:10.1007/BF01031095]

Hoek, E., Brown, E.T., 1980. Underground Excavations in Rock. Institute of Mining and Metallurgy, London, UK, p.14-38.

Huang, F., Yang, X.L., 2011. Upper bound limit analysis of collapse shape for circular tunnel subjected to pore pressure based on the Hoek-Brown failure criterion. Tunnelling and Underground Space Technology, 26(5):614-618. [doi:10. 1016/j.tust.2011.04.002]

Huang, F., Yang, X.L., Zhao, L.H., 2012. Upper bound solution of supporting pressure for a shallow square tunnel based on the Hoek-Brown failure criterion. Journal of Zhejiang University-SCIENCE A (Applied Physics \& Engineering), 13(4):284-292. [doi:10.1631/jzus.A1100246]

Li, H., 2013. Instability Mode and Corresponding Evolution Law of the Ground with Voids Induced by Shallow Tunnelling. MS Thesis, Beijing Jiaotong University, Beijing, China (in Chinese).

Meng, Y., Lei, M.T., Lin, Y.S., et al., 2012. Models and mechanisms of drilling-induced sinkhole in China. Environmental Earth Sciences, 67(7):1961-1969. [doi:10. 1007/s12665-012-1637-8]

Miao, X.X., Li, S.C., Chen, Z.Q., 2009. Bifurcation and catastrophe of seepage flowsystem in broken rock. Mining
Science and Technology, 19(1):1-7. [doi:10.1016/S16745264(09)60001-6]

Pan, Y., Zhang, Y., Yu, G.M., 2006. Mechanism and catastrophe theory analysis of circular tunnel rockburst. Applied Mathematics and Mechanics (English Edition), 27(6): 841-852. [doi:10.1007/s10483-006-0615-y]

Protodyakonov, M.M., 1907. Rock Pressure on Mine Support (Theory of Mine Support). Tipografiya Gubernskogo Zemstva, Yekaterinoslav, p.23-45 (in Russian).

Qiu, Y.L., Zhang, X.X., Shi, B.T., et al., 2012. Checking analysis of lining structural strength in aeolian sand tunnel. Advanced Materials Research, 382:307-311. [doi:10. 4028/www.scientific.net/AMR.382.307]

Ren, S., Wang, Z., Jiang, D.Y., et al., 2013. Study on the catastrophe model of the surrounding rock and simulating the constructing process by DEM in Gonghe tunnel. Third International Conference on Intelligent System Design and Engineering Applications, Hong Kong, China. IEEE, Piscataway, USA, p.1355-1357. [doi:10.1109/ISDEA. 2012.321]

Tao, Y., Cao, J., Hu, J.M., et al., 2013. A cusp catastrophe model of mid-long-term landslide evolution over low latitude highlands of China. Geomorphology, 187:80-85. [doi:10.1016/j.geomorph.2012.12.036]

Terzaghi, K., 1946. Rock Defects and Loads on Tunnel Supports. Harvard University Press, Massachusetts, USA, p.17-99.

Thom, R., 1972. Structural Stability and Morphogenesis. Westview Press, Colorado, USA, p.1-400.

Wang, G.Q., Wang, T., 2007. Calculus of Variations and Its Application in Solid Mechanics. China Railway Publishing House, Beijing, China, p.3-30 (in Chinese).

Xia, C.C., Xu, C.B., Zhao, X., 2012. Study of the strength reduction DDA method and its application to mountain tunnel. International Journal of Computational Methods, 9(3):1250041. [doi:10.1142/S0219876212500417]

\section{中文碳要:}

本文题目: 基于霍克-布朗准则的深埋隧道塌方机制泛函突变分析

Functional catastrophe analysis of collapse mechanisms for deep tunnels based on the Hoek-Brown failure criterion

研究目的: 分析深埋隧道的塌方机制并推导塌方体形状曲线的解析表达式。

创新要点: 基于霍克-布朗准则, 采用泛函突变理论推导得到了深埋隧道塌方体形状曲线的解析表达式, 并据此分析了围岩参数变化对塌方体形状的影响规律。

研究方法: 通过理论分析建立深埋圆形隧道的解析模型（图 1), 采用泛函突变理论推导基于霍克-布朗准 则的隧道塌方体形状曲线解析表达式, 并研究围岩参数变化对塌方体形状的影响规律 (图 3 和 4, 表 2)。通过与自然拱理论和模型试验结果的对比（图 5-7）验证本文解析解的正确性。

重要结论: 采用泛函突变理论推导了基于霍克-布朗准则的深埋隧道塌方体形状曲线解析表达式。该解析 表达式简洁直观，不仅可以预测无支护条件下隧道的塌落体尺寸，还可以估算塌落围岩作用 于衬砌上的荷载。

关键词组: 深埋隧道; 隧道塌方; 泛函突变理论; 霍克-布朗准则 